\section{Adjuvant Etoposide Plus Cisplatin for Patients With Pathologic Stage II Nonseminomatous Germ Cell Tumors: Is This the Preferred Option?}

\section{TO THE EDITOR:}

Cisplatin-based combination chemotherapy for the treatment of testicular cancer has resulted in a transformational change in outcomes, resulting in cures in $80 \%$ of patients with advanced disease. During ASCO's 50-year anniversary, it was recognized as one of the five leading advances in cancer research. ${ }^{1}$ All medical oncologists are aware of the high success rate with modern-day chemotherapy, making metastatic testis cancer the most chemocurative malignancy. Surgical treatment of enlarged abdominal lymph nodes by skilled and experienced urologic surgical oncologists remains an important cornerstone in locoregional disease. There is no solid tumor that has a higher cure rate with nodal resection than testis cancer. With retroperitoneal lymph node dissection (RPLND), these excellent cure rates are influenced by the skill and experience of the medical oncologists and urologists at tertiary centers. ${ }^{2}$

The management of any solid tumor with spread to regional lymph nodes almost always involves postoperative adjuvant chemotherapy to ensure an optimal cure rate. However, testis cancer is different. Recurrence can be detected serologically (alphafetoprotein or human chorionic gonadotropin), and the sites are predictably screened with imaging studies. Thus, most patients relapsing after RPLND will have goodrisk metastatic disease with a low amplitude of tumor markers and small-volume metastatic deposits. Between 1979 and 1984, 195 pathologic stage II patients were enrolled in a phase III intergroup study of two courses of adjuvant cisplatin-based chemotherapy versus observation. Relapses after adjuvant chemotherapy were rare, compared with observation, but there was no difference in over-all survival. $^{3}$

In their recent article in Journal of Clinical Oncology, McHugh et $\mathrm{al}^{4}$ updated the Memorial Sloan Kettering results with adjuvant etoposide plus cisplatin. Earlier results were published in two previous papers. ${ }^{5,6}$ They conclude that the inclusion of bleomycin is not necessary in the adjuvant setting. This is not surprising because a significant number of patients will be cured with RPLND alone, and bleomycin is the least effective drug in bleomycin plus etoposide plus cisplatin
(BEP) combination chemotherapy. For patients

high-risk clinical stage I disease (vascular invasion al lu predominant embryonal cell carcinoma), the relapse rate is higher than in those pathologic stage II cases reported by McHugh. ${ }^{4}$ A single course of BEP will achieve a relapse rate of $3 \%$ or less. ${ }^{7}$

Twenty years ago, we published our data with two courses of BEP adjuvant chemotherapy. ${ }^{8}$ We have not updated our results in a larger sample size because we consider observation, not adjuvant BEP or EP, to be the preferred option, including for those patients with N2 disease. Relapse after RPLND will almost uniformly be cured with $\mathrm{BEP} \times 3$, thus making it difficult to justify two courses of adjuvant chemotherapy. However, if an institution prefers EP $\times 4$ rather than BEP $\times 3$ for good-risk metastatic disease, that might alter the equation. The major goal of RPLND is surgi-cal cure, not staging, and also to avoid chemotherapy. Late complications of cisplatin combination chemotherapy have been well described. ${ }^{9}$ Platinum can be detected $\geq 10$ years after administration. Although it is conjectural whether this is responsible for the increased incidence of cardiovascular complications and second malignancies and whether it is applicable for EP $\times 2$, it raises a level of caution about adjuvant chemotherapy. In our opinion, for the majority of patients with pathologic stage II nonseminoma undergoing primary RPLND, surveillance rather than adjuvant chemotherapy is the preferred approach.

\section{Lawrence H. Einhorn, MD; Nabil Adra, MD; and Nasser Hanna, MD}

Indiana University Melvin and Bren Simon Cancer Center, Indianapolis, IN

\section{Craig Nichols, MD}

SWOG Chair's Office and Testicular Cancer Commons, Portland, OR

\section{AUTHORS' DISCLOSURES OF POTENTIAL CONFLICTS OF INTEREST AND DATA AVAILABILITY STATEMENT}

Disclosures provided by the authors and data availability statement (if applicable) are available with this article at DOI https://doi.org/ 10.1200/JC0.20.00702.

\section{REFERENCES}

1. Einhorn LH, Donohue J: Cis-diamminodichloroplatinum, vinblastine, and bleomycin combination chemotherapy in disseminated testicular cancer. Ann Intern Med 87:293-298, 1977

2. Tandstad T, Kollmannsberger CK, Roth BJ, et al: Practice makes perfect: The rest of the story in testicular cancer as a model curable neoplasm. J Clin Oncol 35:3525-3528, 2017

3. Williams SD, Stablein DM, Einhorn LH, et al: Immediate adjuvant chemotherapy versus observation with treatment at relapse in pathological stage II testicular cancer. N Engl J Med 317:1433-1438, 1987 
4. McHugh DJ, Funt SA, Silber D, et al: Adjuvant chemotherapy with etoposide plus cisplatin for patients with pathologic stage II nonseminomatous germ cell tumors. J Clin Oncol 38:1332-1337, 2020

5. Motzer RJ, Sheinfeld J, Mazumdar M, et al: Etoposide and cisplatin adjuvan therapy for patients with pathologic stage II germ cell tumors. J Clin Onco 13:2700-2704, 1995

6. Kondagunta GV, Sheinfeld J, Mazumdar M, et al: Relapse-free and overall survival in patients with pathologic stage II nonseminomatous germ cell cancer treated with etoposide and cisplatin adjuvant chemotherapy. J Clin Oncol 22:464-467, 2004

7. Cullen M, Huddart R, Joffe J, et al: The 111 study: A single arm, phase 3 tria evaluating one cycle of bleomycin, etoposide and cisplatin as adjuvant chemotherapy in high-risk, stage 1 nonseminomatous or combined germ cell tumours of the testis. Eur Urol 77:344-351, 2020

8. Behnia M, Foster R, Einhorn LH, et al: Adjuvant bleomycin, etoposide and cisplatin in pathological stage II non-seminomatous testicular cancer. The Indiana University experience. Eur J Cancer 36:472-475, 2000

9. Sprauten M, Darrah TH, Peterson DR, et al: Impact of long-term serum platinum concentrations on neuro- and ototoxicity in cisplatin-treated survivors of testicular cancer. J Clin Oncol 30:300-307, 2012

DOI: https://doi.org/10.1200/JC0.20.00702; Published at ascopubs.org/ journal/jco on July 7, 2020. 
AUTHORS' DISCLOSURES OF POTENTIAL CONFLICTS OF INTEREST

\section{Adjuvant Etoposide Plus Cisplatin for Patients With Pathologic Stage II Nonseminomatous Germ Cell Tumors: Is This the Preferred Option?}

The following represents disclosure information provided by authors of this manuscript. All relationships are considered compensated unless otherwise noted. Relationships are self-held unless noted. I = Immediate Family Member, Inst = My Institution. Relationships may not relate to the subject matter of this manuscript. For more information about ASCO's conflict of interest policy, please refer to www.asco.org/rwc or ascopubs.org/jco/authors/author-center.

Open Payments is a public database containing information reported by companies about payments made to US-licensed physicians (Open Payments).

Lawrence H. Einhorn

Stock and Other Ownership Interests: Amgen, Biogen

\section{Nabil Adra}

Research Funding: Merck (Inst), Genentech (Inst)

No other potential conflicts of interest were reported. 